\title{
Design and Implement of a Programmable Logic Controller (PLC) for Classical Control Laboratory ${ }^{*}$
}

\author{
Yousif I. Al Mashhadany \\ Electrical Engineering Department, College of Engineering, University of Anbar, Baghdad, Iraq \\ Email: yousif phd@hotmail.com
}

Received October 31, 2011; revised December 1, 2011; accepted December 9, 2011

\begin{abstract}
The design and implementation of a classical control system laboratory based on PLC control system is introduced in this paper. To design and implement the system, two parts must be produced. The first is software for PLC and the second is hardware for experiments related to the programs. The PLC control system used in the design is LS industrial system company GM7-DR40A 24/16 Digital I/O and single Analog I/O module, two photoelectric sensors from Atonic company': the first with the model BR100-DDT-P, and the second BEN10M-TFR. An approximate sensor with 5-sides is detected, four of CMOS BCD-7-Segment driven by CD4511B, two relays: 2-poles and 3-poles, six voltages and an ammeter measurement, DC motor and 24 VDC power supply and many connecters and pinions. Satisfactory results are obtained by executing twenty four experiments for classical control theory that fulfill the requirements of control theory in undergraduate stage and replace the old experiments executed by PID controller where the practice system is implemented by PLC control now.
\end{abstract}

Keywords: PLC Control System; Photoelectric Sensor

\section{Introduction}

PLC's were developed in the late 1960's to eliminate the large cost involved in changing complicated relay based machine control systems. These systems were inflexible. Their major rewiring or complete replacement was necessary every time when the production requirements changed and control sequences had to be modified. It became very expensive when changes were frequent. The idea to replace relay systems with flexible microprocessor based Programmable logic controllers PLC's was a revolutionary step in the industrial control business [1].

PLC's have been used for many years at many applications. They are the main control elements of several subsystems which require relatively slow data monitoring: the radiation control, personal safety, fire and smoke alarm. All these subsystems are very cost effective and reliable. Adding new equipment to control or changing operating conditions to implement new control features can easily be done by the skilled technician staff, without any help from the Controls Software Group.

The software development tool and some ideas of device support for DirectNet PLC's became the basis of a new PLC control software library at control Lab. The software acts as a DirectNet master through any RS-232 port and consists of three main parts: a PLC driver with a

*Implement the PLC as trainer at undergraduate laboratory. state machine control block, a device support module, and a common serial driver [2,3].

Basic PLC's are available on a single printed circuit board as shown in Figure 1. They are sometimes called single board PLC's or open frame PLC's. These are totally self-contained (with the exception of a power supply) and, when installed in a system, they are simply mounted inside a controls cabinet on threaded standoffs.

Screw terminals on the printed circuit board allow for the connection of the input, output, and power supply wires. These units are generally not expandable, meaning that extra inputs, outputs, and memory cannot be added

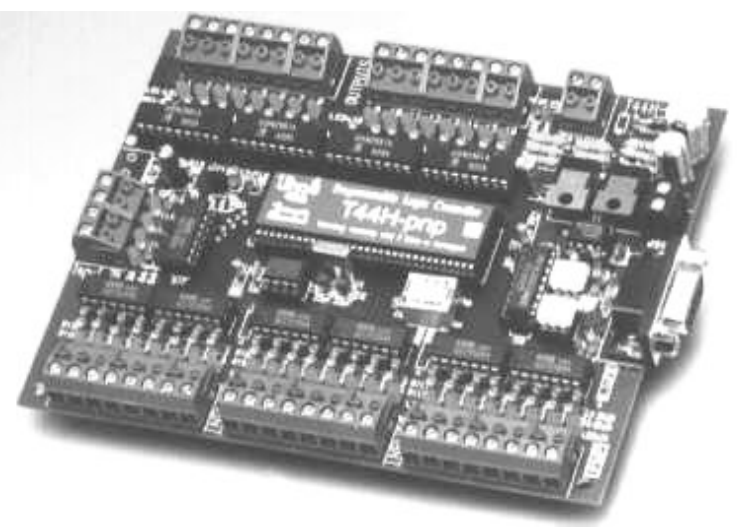

Figure 1. Open frame PLC [4]. 
to the basic unit. However, some of the more sophisticated models can be linked by cable to expansion boards that can provide extra I/O. Therefore, with few exceptions, when using this type of PLC, the system designer must take care to specify a unit that has enough inputs, outputs, and programming capability to handle both the present need of the system and any future modifications that may be required. Single board PLC's are very inexpensive, easy to program, small, and consume little power, but, generally speaking, they do not have a large number of inputs and outputs, and have a somewhat limited instruction set. They are best suited to small, relatively simple control applications [4].

\section{Hardware Design for the Model}

Inputs to, and outputs from, a PLC are necessary to monitor and control a process. Both inputs and outputs can be categorized into two basic types: logical or continuous. Consider the example of a light bulb. If it can only be turned on or off, it is logical control. If the light can be dimmed to different levels, it is continuous. Continuous values seem more intuitive, but logical values are preferred because they allow more certainty, and simplify control. As a result most controls applications (and PLC's) use logical inputs and outputs for most applications [5].

Outputs to actuators allow a PLC to cause something to happen in a process. A short list of popular actuators is given below in order of relative popularity.

- Solenoid Valves-logical outputs that can switch a hydraulic or pneumatic flow.

- Motor Starters - motors often draw a large amount of current when started, so they require motor starters, which are basically large relays.

- Servo Motors - a continuous output from the PLC can command a variable speed or position.

Outputs from PLC's are often relays, but they can also be solid state electronics such as transistors for DC outputs or Triacs for AC outputs. Continuous outputs require special output cards with digital to analog converters, or can be used another module for PLC analogue o/p.

Inputs come from sensors that translate physical phenomena into electrical signals. Typical examples of sensors are listed below in relative order of popularity.

- Proximity Switches - use inductance, capacitance or light to detect an object logically.

- Switches - mechanical mechanisms will open or close electrical contacts for a logical signal.

- Potentiometer-measures angular positions continuously, using resistance.

- LVDT (linear variable differential transformer) - measures linear displacement continuously using magnetic coupling.

- Photoelectric sensor use the ultrasonic signal to detect any interruption within limited range.

Inputs for a PLC come in a few basic varieties; the simplest are AC and DC inputs. Sourcing and sinking inputs are also popular. This output method dictates that a device does not supply any power. Instead, the device only switches current on or off, like a simple switch [6].

PLC inputs must convert a variety of logic levels to the 5VDC logic levels used on the data bus. This can be done with circuits similar to those shown in Figure 2. Basically the circuits condition the input to drive an optocoupler. This electrically isolates the external electrical circuitry from the internal circuitry. Other circuit components are used to guard against excess or reversed voltage polarity.

PLC outputs must convert the 5VDC logic levels on the PLC data bus to external voltage levels. This can be done with circuits similar to those shown in Figure 3. Basically, the circuits use an optocoupler to switch external circuitry. This electrically isolates the external electrical circuitry from the internal circuitry. Other circuits components are used to guard against excess or reversed voltage polarity [7].

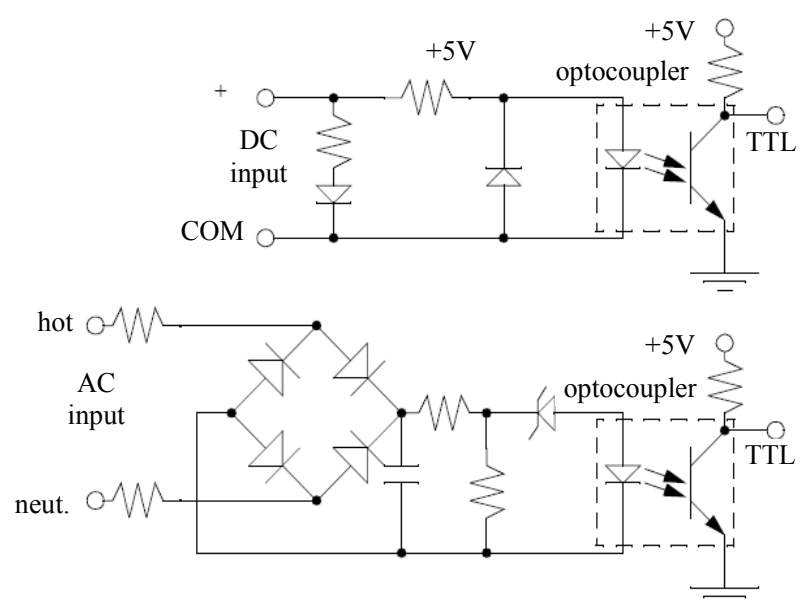

Figure 2. PLC input circuits [6].
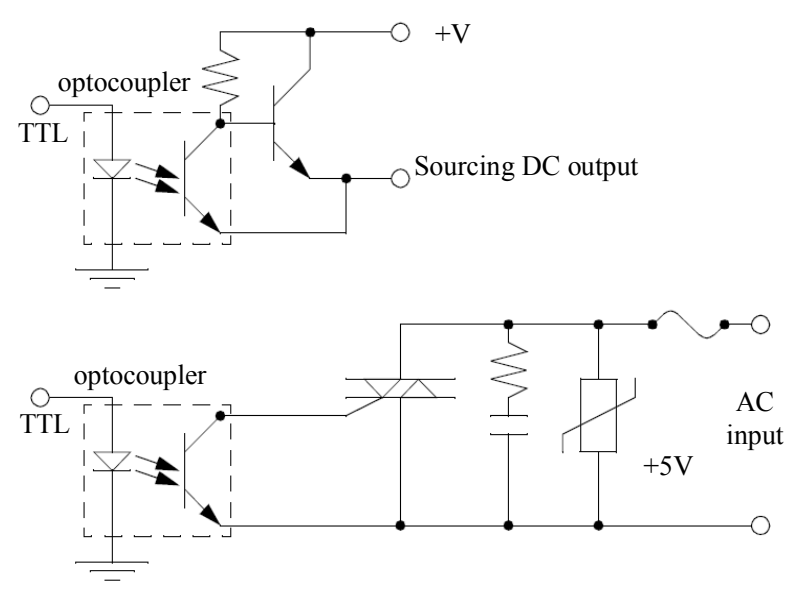

Figure 3. PLC output circuits [6]. 
The overall design of the model is shown in Figure 4. and the schematic of this design shown in Figure 5. The full description of connection for this design is very complex and it has many details, therefore I will describe the connection of the main parts only.

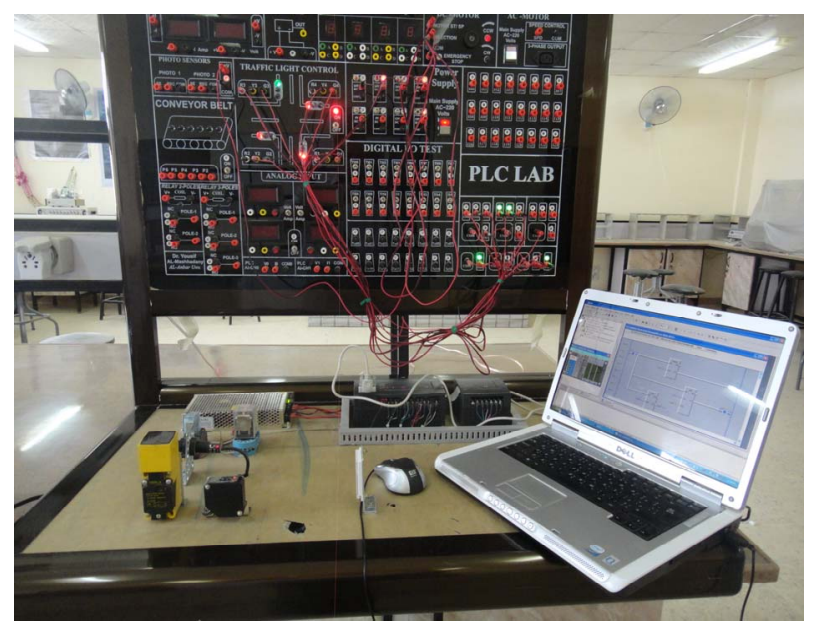

Figure 4. Classical control system laboratory based on PLC.

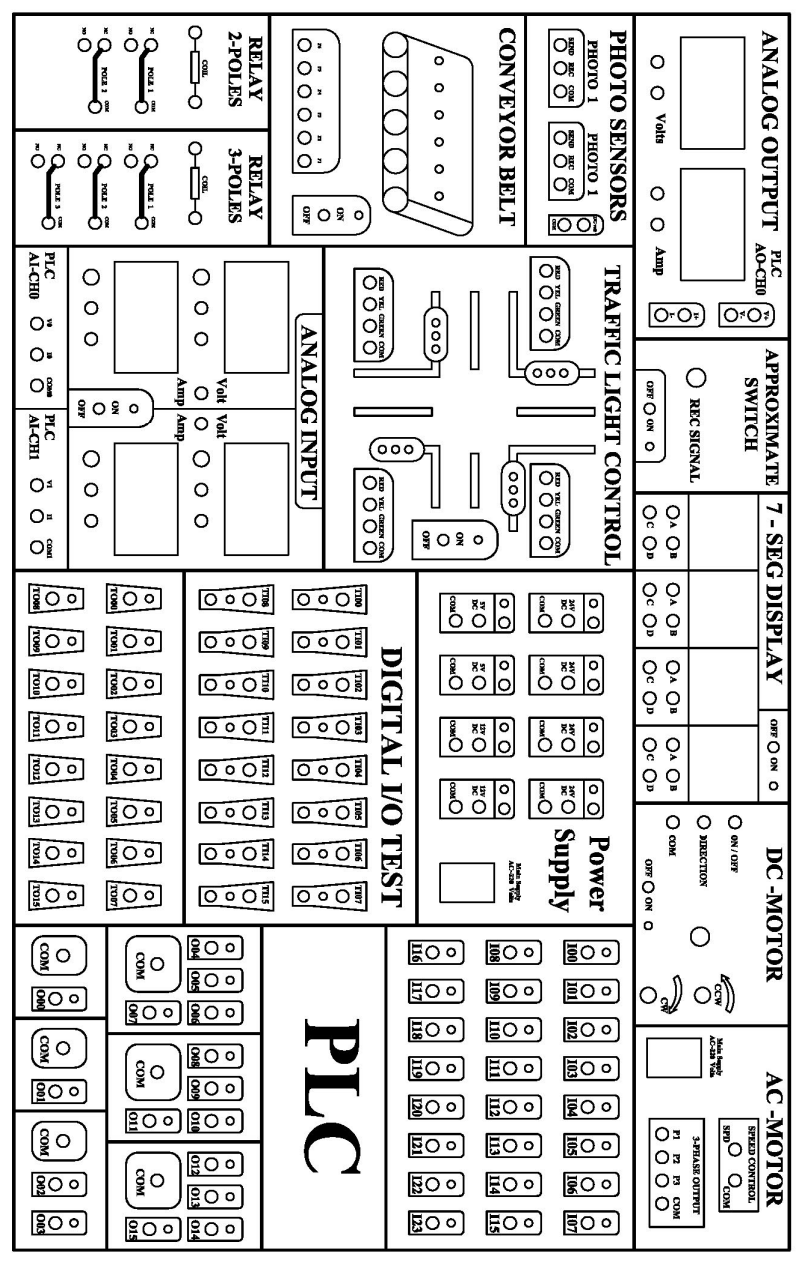

Figure 5. Schematic of the lab.

\section{Hardware Design of PLC Trainer Model}

The hardware design of this trainer consists of three main parts:

- Main unit of PLC model and its power supply

The PLC model used in this design contains two parts. The first presents the main digital PLC that contains: RS232 connecter to apply the software program to PLC, 24 digital inputs drawing on cover by $\mathrm{I00} \rightarrow \mathrm{I} 23,16$ digital outputs drawing by $\mathrm{O} 00 \rightarrow$ O15with six common ports and 25-pins adapter to execute the additional modules of PLC. Figure 6 shows the main unit of PLC with power supply.

The second part of this unit is a single analog PLC that contains two channels of analog inputs which are drawing by symbols (CH0(input) (I0,V0,COM0) and $\mathrm{CH} 1$ (input) (I1,V1, COM1)), one analog output channel (CH0(output) $(\mathrm{V}+, \mathrm{V}-, \mathrm{I}+, \mathrm{I}-)$ and 25-pins adapter to add another module (see Figure 6).

Power supply for this unit is $24 \mathrm{VDC} / 5 \mathrm{~A}$ contain two supply ports (see Figure 6). The connection of I/O PLC with board achieved by sets of pinions and LED's for indication (On/Off). Figure 7 presents the connection between PLC unit and in the experiments board.

- Sensor, Relay and other components

The trainer contains two types of sensors; an approximate sensor that has 5-sides detection with model TURCK Bi15-cp40 APcx sn:15 mm and photoelectric sensors with two models BR100-DDT-P and BEN10M-TFR. The first detects any interrupt within a range of 8 meters and the second detects the reflection from a certain crystal surface within a range of 8 meters.

Two types of relays are used in the board: two-poles and three-poles 24VDC. Figure 8 presents the two relays, the sensors and the connection in the board.

Many other components that are used in the broad to connect the experiments such as four 7-segments, three digital ammeters and three digital voltmeter, three variable resistance $20 \mathrm{Kohm}$ to execute the analog to digital convertor (A/D) and opposite (D/A) and power supply with two $12 \mathrm{VDC}$ ports, two $5 \mathrm{VDC}$ ports and four 24 VDC ports. Figure 9 presents the power supply ports in the board. Figure $\mathbf{1 0}$ presents the ammeters and converter $\mathrm{A} / \mathrm{D}$.

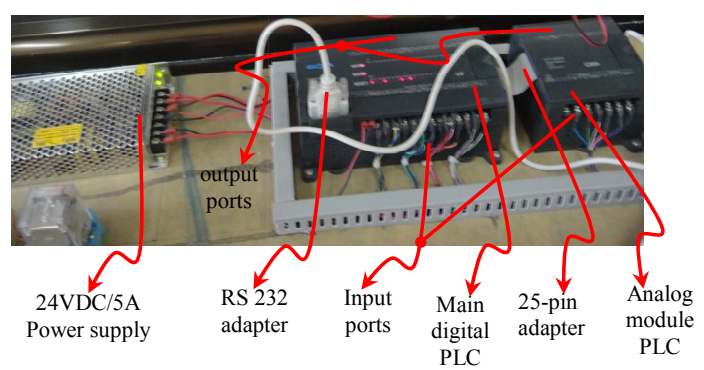

Figure 6. Main PLC unit with power supply. 


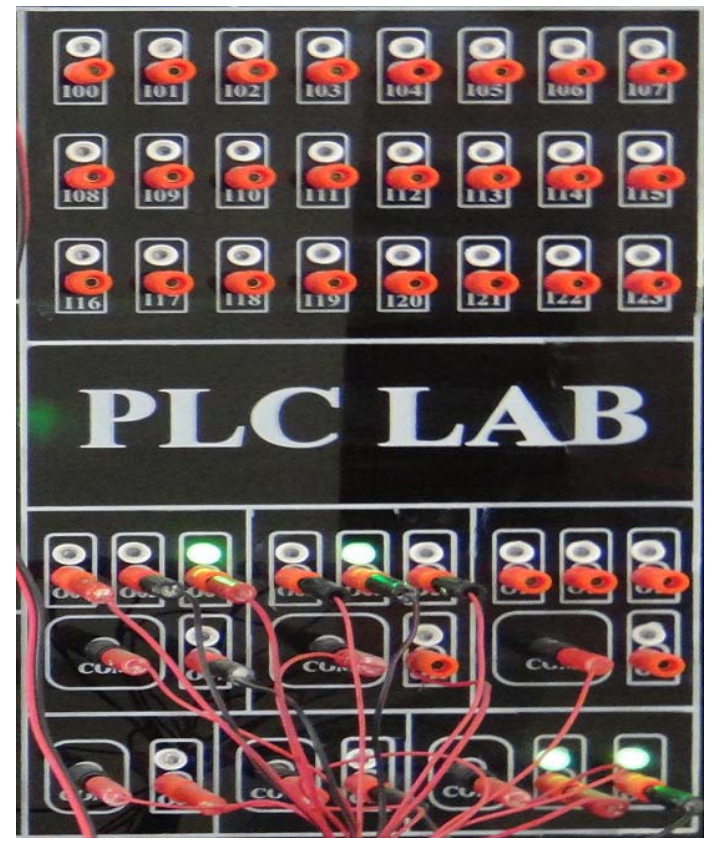

Figure 7. PLC connection with experiments board.

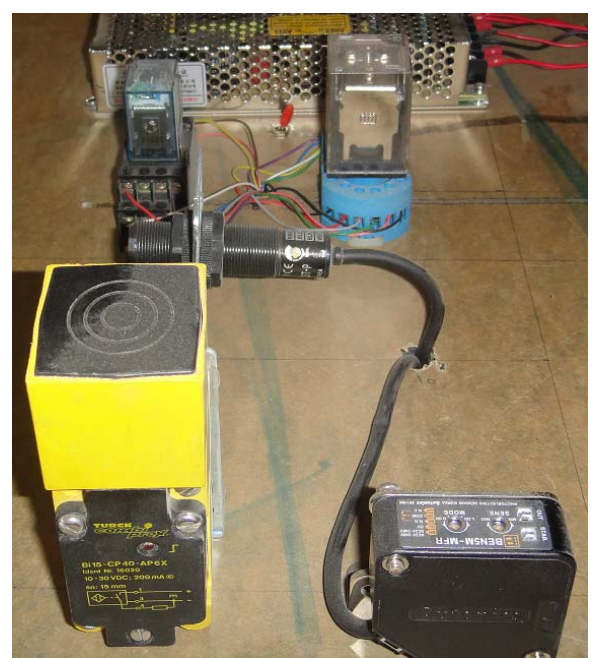

(a)

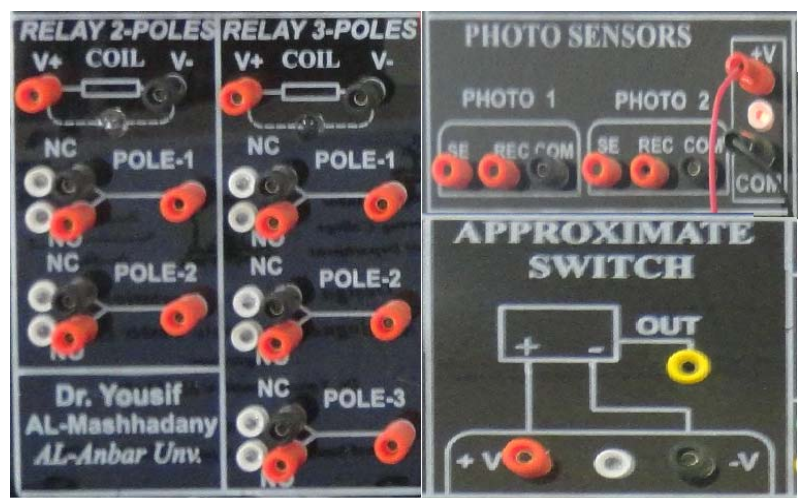

(b)

Figure 8. Relay and sensors and its connection in the board.

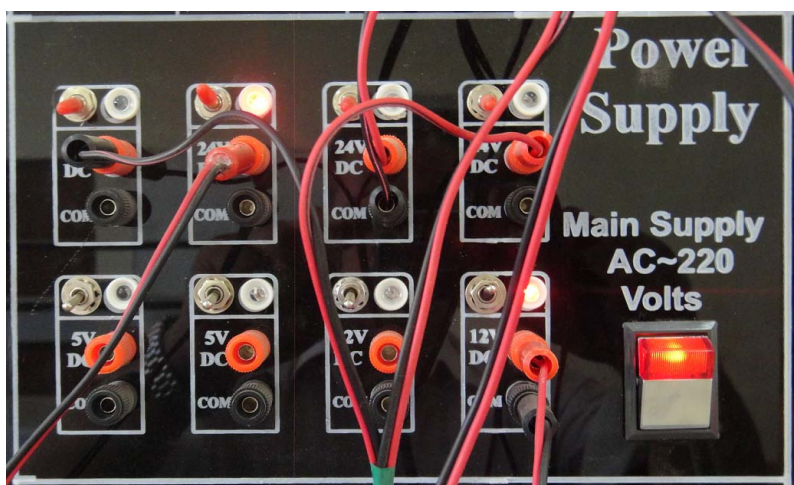

Figure 9. Power supply connection in the board.

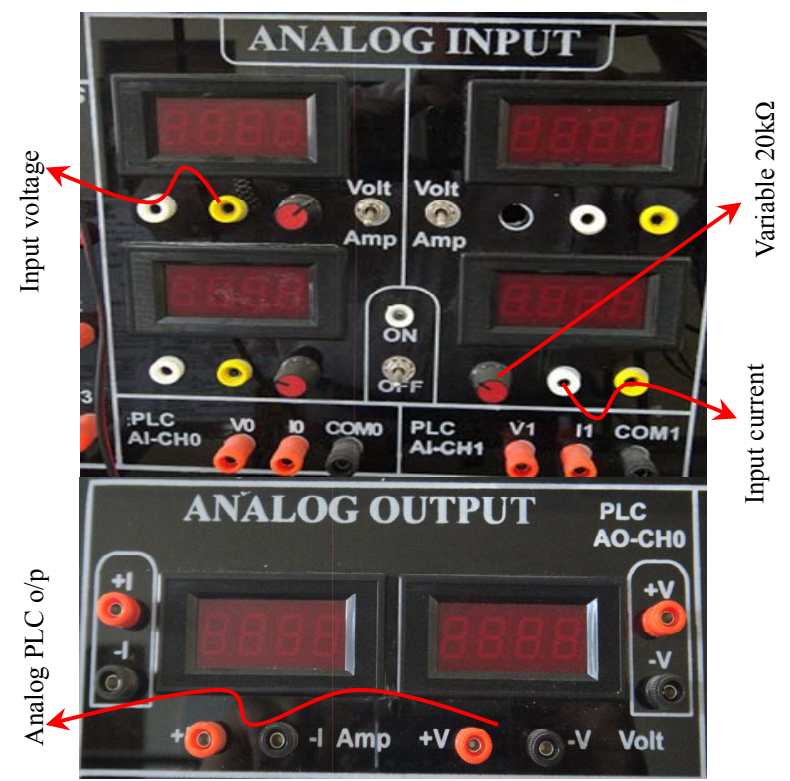

Figure 10. Ammeters and varying for A/D.

DC motor with model GMN-3MX027A DC24V and driver for $\mathrm{AC}$ and $\mathrm{DC}$ motor start/stop, direction and emergency control of DC motor and speed control AC motor are implemented in the board. Figure 11 presents the connection of the motor and drivers in the board.

- Experiments board

The board is designed to implement many experiments, some of the experiments the connection diagram is plotted on the board such as traffic light control, I/O PLC test DC motor and 7-segemnts. Figure 12 presents the overall board design.

\section{Software Design of PLC Trainer Model}

LS industrial system company GM7-DR40 PLC uses the language announced as an international standard by IEC (International Electro technical Commission). The international standard languages such as LD, SFC, IL available to select the language are easy to apply to the system. I used the LD (Ladder Diagram) to build the program for 
experiments. Figure 13 presents the main window for building the program and simulating the instruction before writing on the memory of PLC through the RS232 connecter.

Every instruction in the program must be compiled before writing the process code to the memory of PLC. After compilation we can simulate the program to check and explain the working of real PLC with this program and the input to simulation process can be done manually, all these processes are shown in Figure 13.

\section{Experiments of PLC Trainer}

This trainer is designed to implement the experiments of classical control laboratory to replace the PID controller

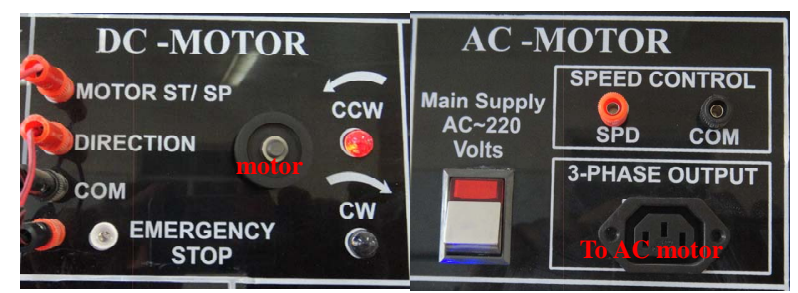

Figure 11. DC and AC motor drivers' connection.

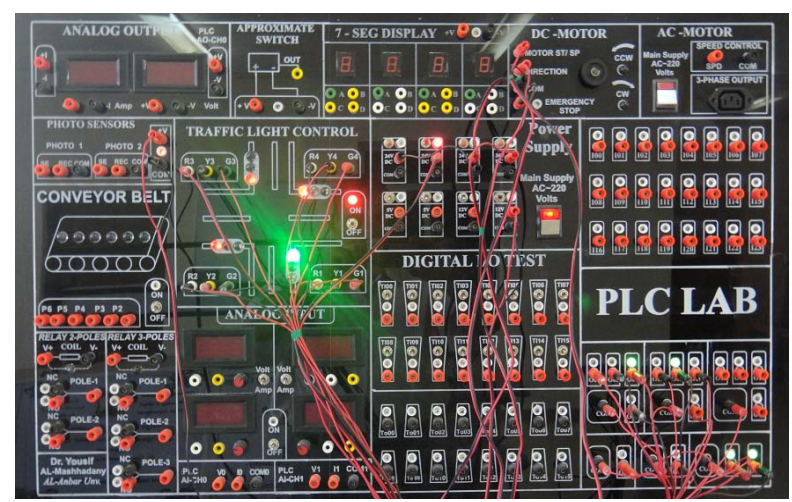

Figure 12. Overall board design.

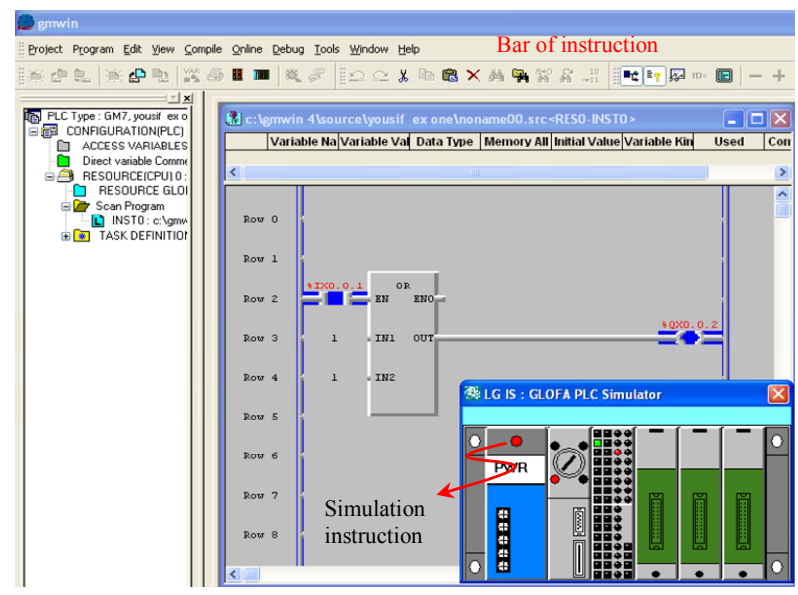

Figure 13. Main LD program and its simulation. by PLC controller where the last has a wide range in industrial application. The experiments that are implemented in this lab can be divided into three main groups according to application field in classical control approach: Logic process, power system and interactive process control, where by using this trainer six experiments can be done in logic process, ten experiments in power system and eight experiments in the interactive process control. Every experiment has a procedure for connection on the board and program for writing with PLC memory and then run the connection. For example, the implementation of traffic light control can be discussed by the procedure as follows:

1) Switch OFF main supply 220VAC \& switch OFF 24VDC.

2) Connect (24VDC) from Power supply to (COM) of (O00 ‥ O11).

3) Connect (O00 $\cdots \mathrm{O} 11)$ to traffic light diagram on the board according to the diagram that is shown in Table 1.

4) Switch ON main supply 220VAC.

5) Download the ladder program of traffic light control to PLC. The program is similar to the program that is shown in previous page (Figure 13).

Table 1. Connection diagram of traffic light (experiment).

\begin{tabular}{cccccc}
\hline No. & $\begin{array}{c}\text { From Port } \\
\text { o/p PLC }\end{array}$ & $\begin{array}{c}\text { To Port } \\
\text { on board }\end{array}$ & No. & $\begin{array}{c}\text { From Port } \\
\text { o/p PLC }\end{array}$ & $\begin{array}{c}\text { To Port } \\
\text { on board }\end{array}$ \\
\hline 1 & O00 & R1 & 7 & O06 & R3 \\
2 & O01 & Y1 & 8 & O07 & Y3 \\
3 & O02 & G1 & 9 & O08 & G3 \\
4 & O03 & R2 & 10 & O09 & R4 \\
5 & O04 & Y2 & 11 & O10 & Y4 \\
6 & O05 & G2 & 12 & O11 & G4 \\
\hline
\end{tabular}

Table 2. Sequence of light $\mathrm{ON}$ in traffic display.

\begin{tabular}{|c|c|c|c|c|c|}
\hline No. & Time & Traffic 1 & Traffic 2 & Traffic 3 & Traffic 4 \\
\hline \multirow{2}{*}{ State 1} & 0 to $10 \mathrm{sec}$ & Green 1 & Red 2 & Red 3 & Red 4 \\
\hline & 11 to $15 \mathrm{sec}$ & Yellow 1 & Red 2 & Red 3 & Red 4 \\
\hline \multirow{2}{*}{ State 2} & 16 to $25 \mathrm{sec}$ & Red 1 & Green 2 & Red 3 & Red 4 \\
\hline & 31 to $40 \mathrm{sec}$ & Red 1 & Yellow 2 & Red 3 & Red 4 \\
\hline \multirow{2}{*}{ State } & 41 to $45 \mathrm{sec}$ & Red 1 & Red 2 & Green 3 & Red 4 \\
\hline & 46 to $50 \mathrm{sec}$ & Red 1 & Red 2 & Yellow 3 & Red 4 \\
\hline \multirow{2}{*}{ State } & 51 to $60 \mathrm{sec}$ & Red 1 & Red 2 & Red 3 & Green 4 \\
\hline & 61 to $65 \mathrm{sec}$ & Red 1 & Red 2 & Red 3 & Yellow 4 \\
\hline Return to & 66 to $75 \mathrm{sec}$ & Green 1 & Red 2 & Red 3 & Red 4 \\
\hline and so on... & 76 to $80 \mathrm{sec}$ & Yellow 1 & Red 2 & Red 3 & Red 4 \\
\hline
\end{tabular}


6) Switch ON 24VDC, the traffic light LEDs must be activates according the sequence at is shown in Table 2. Note: the value of time in this experiment is taken fast to explain all process in short time.

The practice connection for this experiment is shown in the previous page (Figure 12).

\section{Conclusions}

The practice results that are obtained in real implementation of all experiments in the laboratory and the simulation results by LD program, it can be seen that the implementation of PLC controller with classical control systems is necessary. This implementation has high performance, high accuracy and more speed response compared to the classical controller.

The trainer presents practice simulation for many real systems; therefore it is very suitable for classical laboratory unit with undergraduate students.

\section{Acknowledgements}

Special thanks are due to AL Anbar University/Engineering College for support me with the implementation of the PLC trainer laboratory.

\section{REFERENCES}

[1] P. Chevtsov, S. Higgins, S. Schaffner and D. Seidman, "PLC Support Software at Jefferson Lab," Jefferson Lab, Newport News, 2002.

[2] J. R. Hackworth and F. D. Hackworth, "Programmable Logic Controllers: Programming Methods and Applications," Prentice Hall, Upper Saddle River, p. 13.

[3] L. A. Bryan and E. A. Bryan, "Programmable Controllers Theory and Implementation," 2nd Edition, Industrial Text Company Publication, Atlanta, 1997.

[4] H. Jack, "Automating Manufacturing Systems with PLCs, Version 4.7," Copyright (c) 1993-2005 Hugh Jack, 2005.

[5] S. Yurkovich and K. M. Passino, "A Laboratory Course on Fuzzy Control," IEEE Transactions on Education, Vol. 42, No. 1, 1999, pp. 15-21.

[6] A. Sirinterlikci, "Development of a Comprehensive Industrial Controls Course in a Manufacturing Engineering Program," American Society for Engineering Education, Washington DC, 2006.

[7] X. Yu, X. Feng, C. Xiong and S. Jiaotong, "The Design and Implementation of Elevator Group Control System Research Platform," Proceedings of the 2009 International Workshop on Information Security and Application, Qingdao, 21-22 November 2009. 